\title{
Dapagliflozin in patients with type 2 diabetes mellitus
}

\author{
Theodosios D. Filippatos, Evangelos N. Liberopoulos and Moses S. Elisaf
}

\begin{abstract}
Dapagliflozin is a selective and reversible inhibitor of sodium-glucose linked transporter type 2 (SGLT2), which mediates approximately $90 \%$ of active renal glucose reabsorption in the early proximal tubule of the kidney. Dapagliflozin significantly reduces glucose reabsorption and decreases serum glucose concentration in an insulin-independent manner. The decrease of glucose reabsorption by dapagliflozin has also been associated with a reduction in body weight. Furthermore, the drug modestly reduces blood pressure levels through weight loss and its action as osmotic diuretic. Dapagliflozin has been approved as monotherapy in patients with type 2 diabetes mellitus (T2DM) who cannot tolerate metformin or in combination with other antidiabetic drugs, with the exception of pioglitazone due to the theoretical increased risk of bladder cancer. The drug should not be prescribed in patients with moderate or severe renal impairment or in patients at risk for developing volume depletion. Dapagliflozin is associated with increased incidence of genital and lower urinary tract infections, but these infections are usually mild to moderate and respond to standard antimicrobial treatment. Based on current evidence, dapagliflozin is a useful drug for patients with T2DM with a favorable safety profile. However, further research regarding the effects of dapagliflozin on cardiovascular outcomes is needed.
\end{abstract}

Keywords: adverse effects, blood pressure, body weight, dapagliflozin, diabetes mellitus, drug interactions, metabolic effects, sodium-glucose linked transporter type 2 inhibitors

\section{Introduction}

Type 2 diabetes mellitus (T2DM) has increasing incidence and is strongly linked with many cardiovascular and renal complications [Leong et al. 2013; American Diabetes Association, 2014]. Different classes of drugs are used in the treatment and prevention of complications in patients with T2DM [Filippatos and Elisaf, 2010a; Ussher et al. 2012; Agouridis et al. 2013; Filippatos and Elisaf, 2013a; Sheikh-Ali et al. 2013; Filippatos et al. 2014]. Current guidelines propose metformin as the preferred initial pharmacological agent for T2DM [American Diabetes Association, 2014]. However, many patients do not achieve the glycemic goal or do not tolerate metformin and the addition of a second oral agent, a glucagonlike peptide 1 (GLP-1) receptor agonist or insulin, is usually needed. Available oral agents, besides metformin, include sulphonylureas, thiazolidinediones, dipeptidyl peptidase 4 (DPP-4) inhibitors and the newer selective inhibitors of sodium-glucose linked transporter type 2 (SGLT2).
Dapagliflozin is a selective SGLT2 inhibitor and has been recently approved for the treatment of patients with T2DM. The aim of this review is to present the available evidence regarding the pharmacokinetic properties, the effects on metabolic variables and the adverse effects of dapagliflozin.

\section{Mechanism of action}

SGLT2 mediates approximately $90 \%$ of active renal glucose reabsorption in the S1 segment of early proximal tubule of the kidney [Vallon et al. 2011]. Dapagliflozin is a selective and reversible inhibitor of SGLT2, leading to a significant reduction in glucose reabsorption and decrease of serum glucose concentration in an insulin-independent manner [Balakumar et al. 2014]. Dapagliflozin also improves insulin sensitivity but enhances endogenous glucose production in patients with T2DM [Merovci et al. 2014; Mudaliar et al. 2014]. The reduction in glucose reabsorption by dapagliflozin has been associated
Ther Adv Endocrinol

Metab

2015, Vol. 6(1) 29-41

DOI: $10.1177 /$

2042018814558243

(c) The Author(s), 2014. Reprints and permissions: http://www.sagepub.co.uk/ journalsPermissions.nav

Correspondence to: Moses S. Elisaf,Professor of Internal Medicine FASA, FISA

Department of Internal Medicine, School of Medicine, University of loannina, 45110 loannina, Greece egepiacc.uoi.gr Theodosios D. Filippatos, MD, PhD

Evangelos N. Liberopoulos, Assistant Professor of Internal Medicine Department of Internal Medicine, School of Medicine, University of loannina, Ioannina, Greece 
with a reduction in body weight possibly owing to the decrease of body calories. Furthermore, the drug has been shown to reduce blood pressure levels through its action as an osmotic diuretic and the associated body weight reduction [Oliva and Bakris, 2014].

\section{Clinical pharmacology}

Dapagliflozin is an orally active, reversible and highly selective SGLT2 inhibitor, given in oncedaily doses [Komoroski et al. 2009; Obermeier et al. 2010]. Dapagliflozin has an absolute bioavailability of $78 \%$ in humans [Boulton et al. 2013]. It is rapidly absorbed with a time to maximum plasma concentration of $0.5-1.3 \mathrm{~h}$ [Kasichayanula et al. 2011a]. Dapagliflozin has extensive extravascular distribution, with a mean volume of distribution of 118 liters [Kasichayanula et al. 2014]. Body weight, age, race, sex, or presence of T2DM do not meaningfully affect the exposure of dapagliflozin [Kasichayanula et al. 2014]. However, owing to the absence of evidence, initiation of dapagliflozin therapy is not recommended in patients older than 75 years or less than 18 years [European Commission, 2012]. Food does not meaningfully affect the pharmacokinetics and the efficacy of dapagliflozin [Kasichayanula et al. 2011d].

Dapagliflozin is metabolized by uridine diphosphate glucuronosyltransferase 1A9 (glucuronidation) in the liver and the kidney to a major, inactive metabolite (dapagliflozin 3-O-glucuronide, D3OG), which is mainly cleared via the kidney [Kasichayanula et al. 2014]. Hepatic impairment affects the plasma concentration of dapagliflozin. A study using a single $10 \mathrm{mg}$ oral dose of the drug showed that, compared with healthy subjects, mean maximum plasma concentration of dapagliflozin was $12 \%$ lower and $12 \%$ and $40 \%$ higher in subjects with mild, moderate or severe hepatic impairment, respectively [Kasichayanula et al. 2011c]. No dosage adjustment of the drug is necessary for patients with mild or moderate hepatic impairment. However, clinicians should be cautious when administrating dapagliflozin in patients with severe hepatic impairment, in whom a starting dose of $5 \mathrm{mg}$ is recommended which could be increased to $10 \mathrm{mg}$ if it is well tolerated [European Commission, 2012].

Dapagliflozin and its inactive metabolite are mainly cleared via the kidney [Kasichayanula et al. 2014]. Renal impairment is a major factor for the metabolism and efficacy of dapagliflozin. A study showed that the maximum plasma concentration of dapagliflozin was increased by $4 \%, 6 \%$ and $9 \%$ and D3OG by $20 \%, 37 \%$ and $52 \%$, respectively, in patients with mild, moderate and severe renal impairment compared with those with normal kidney function. However, the pharmacodynamic effects of dapagliflozin were significantly decreased and resulted in reduced efficacy in patients with renal impairment [Kasichayanula et al. 2013b]. Dapagliflozin is indicated in patients with mild renal impairment, but it should not be given in patients with moderate to severe renal impairment (creatinine clearance $<60 \mathrm{ml} / \mathrm{min}$ or estimated glomerular filtration rate $<60 \mathrm{ml} /$ $\mathrm{min} / 1.73 \mathrm{~m}^{2}$ ) [European Commission, 2012].

\section{Therapeutic use of dapagliflozin in patients with T2DM}

\section{Monotherapy}

A phase III, double-blind, placebo-controlled study randomized 282 treatment-naive patients with T2DM [glycosylated hemoglobin (HbA1c) $\geqslant 7.0$ and $\leqslant 10.0 \%$ ] to dapagliflozin $1,2.5$ or $5 \mathrm{mg} /$ day or placebo for 24 weeks (Table 1) [Bailey et al. 2012]. The primary efficacy endpoint was the change in $\mathrm{HbAlc}$ from baseline. Dapagliflozin treatment led to a significant reduction in $\mathrm{HbA} 1 \mathrm{c}(-0.68 \%$ with $1 \mathrm{mg},-0.72 \%$ with $2.5 \mathrm{mg},-0.82 \%$ with $5 \mathrm{mg}$ ) compared with placebo $(+0.02 \%, p<0.0001)$. In a similar manner, dapagliflozin reduced fasting plasma glucose levels and body weight significantly more than placebo ( $p<0.02$ and $p<0.003$, respectively). The significantly greater effects of dapagliflozin resulted in lower rates of addition of rescue medication or discontinuation due to poor glycemic control. Adverse effects were generally similar among treatment groups [Bailey et al. 2012].

Another 24-week, double-blind trial randomized 485 patients with T2DM to placebo or dapagliflozin $2.5,5$ or $10 \mathrm{mg}$ once daily in the morning (main cohort) or evening (exploratory cohort) [Ferrannini et al. 2010]. In the main cohort, mean HbA1C changes from baseline at week 24 were significantly greater with dapagliflozin $(-0.58 \%$, $-0.77 \%,-0.89 \%$ with $2.5,5$ and $10 \mathrm{mg}$, respectively) compared with placebo $(-0.23 \%, p<0.001$ versus 5 and $10 \mathrm{mg}$ ). Similar results were observed in the exploratory evening dose cohort. In patients with high HbA1c at enrolment (10.1-12.0\%, $n=73$ ), dapagliflozin administration induced numerically greater reductions in $\mathrm{HbA} 1 \mathrm{C}$ compared with those observed in patients with lower 
baseline HbA1c levels ( $-2.88 \%$ with $5 \mathrm{mg}$ and $-2.66 \%$ with $10 \mathrm{mg}$ ) [Ferrannini et al. 2010].

The effects of the drug on blood pressure were investigated in a randomized, double-blind trial which randomized 75 patients with T2DM for 12 weeks to dapagliflozin $10 \mathrm{mg} /$ day, hydrochlorothiazide $25 \mathrm{mg} /$ day or placebo [Lambers Heerspink et al. 2013]. The $24 \mathrm{~h}$ ambulatory mean systolic blood pressure (SBP) decreased by $-3.3 \mathrm{mmHg}$ with dapagliflozin [95\% confidence interval (CI) -6.8 to $+0.2 \mathrm{mmHg}$ ], $-6.6 \mathrm{mmHg}$ with hydrochlorothiazide ( $95 \% \mathrm{CI}-9.9$ to $-3.2 \mathrm{mmHg}$ ) and $-0.9 \mathrm{mmHg}$ with placebo $(95 \% \mathrm{CI}-4.2$ to $+2.4 \mathrm{mmHg}$ ) (all values are adjusted for baseline SBP). The greater effect of dapagliflozin compared with placebo was observed during daytime SBP but not during night-time SBP. Dapagliflozin also resulted in a greater decrease in in-office SBP by $-12.3 \mathrm{mmHg}(95 \% \mathrm{CI}-17.8$ to $-6.8 \mathrm{mmHg}$ ) compared with hydrochlorothiazide $[-1.1 \mathrm{mmHg}$ (95\% CI -2.2 to $0.0 \mathrm{mmHg}$ )] or placebo [ -0.1 $\mathrm{mmHg}(95 \% \mathrm{CI}-10.8$ to $+0.6 \mathrm{mmHg})]$. The effects of dapagliflozin or hydrochlorothiazide were independent from concurrent antihypertensive drug use. In a substudy of 30 patients, dapagliflozin treatment resulted in a reduction in plasma volume by $-7.3 \%$ [median (interquartile range) $-12.4 \%$ to $-4.8 \%$ ] compared with hydrochlorothiazide $[+2.8 \%(-10.6 \%$ to $+25.7 \%)]$ or placebo [+5.2\% ( -2.5 to +8.7$)]$. Dapagliflozin treatment resulted in a greater decrease in glomerular filtration rate $[-10.8 \%(95 \% \mathrm{CI}-14.6 \%$ to $-6.7 \%)]$ compared with hydrochlorothiazide $[-3.4 \%$ $(-7.3 \%$ to $+0.6 \%)]$ or placebo $[-2.9 \%(-6.9 \%$ to $+1.2 \%$ )] [Lambers Heerspink et al. 2013].

\section{Addition to metformin}

A double-blind trial randomized 546 patients with T2DM, who had inadequate glycemic control despite receiving metformin, to dapagliflozin $2.5,5$ or $10 \mathrm{mg} /$ day or placebo for 24 weeks (Table 1). Dapagliflozin administration resulted in greater reductions in $\mathrm{HbAlc}(-0.67 \%$ with 2.5 $\mathrm{mg},-0.70 \%$ with $5 \mathrm{mg}$ and $-0.84 \%$ with $10 \mathrm{mg}$ ) compared with placebo $(-0.30 \%$, all $p<0.001)$. Fasting plasma glucose concentration and body weight were also reduced significantly more with dapagliflozin compared with placebo. Adverse effects were generally similar between groups but genital infections occurred more frequently with dapagliflozin [Bailey et al. 2010].

In a long-term extension of the above trial (78 weeks, total 102 weeks), dapagliflozin treatment led to a significant reduction in $\mathrm{HbA} 1 \mathrm{c}$ $(-0.48 \%$ with $2.5 \mathrm{mg},-0.58 \%$ with $5 \mathrm{mg}$ and $-0.78 \%$ with $10 \mathrm{mg}$, all $p<0.001$ ), whereas a small increase $(+0.02 \%)$ was observed with placebo [Bailey et al. 2013]. Furthermore, dapagliflozin administration resulted in sustained reductions in fasting plasma glucose and body weight compared with increases of these variables with placebo. Additionally, significant reductions compared with placebo were observed in uric acid concentration. Dapagliflozin was associated with a higher completion rate (68.3-79.8\%) than the placebo group $(63.5 \%)$. Events suggestive of genital infection and urinary tract infection were reported in a higher rate with dapagliflozin compared with placebo [Bailey et al. 2013].

Another 24-week study randomized 182 patients with $\mathrm{T} 2 \mathrm{DM}$ to dapagliflozin $10 \mathrm{mg} /$ day or placebo on top of metformin [Bolinder et al. 2012]. Dapagliflozin resulted in a significantly greater body weight reduction $(-2.96 \mathrm{~kg})$ compared with placebo $(-0.88 \mathrm{~kg}, p<0.0001)$. Interestingly, a greater weight loss was observed in men compared with women ( $p=0.048$ for sex by treatment interaction). Furthermore, dapagliflozin led to a significant placebo-corrected reduction in fatty mass $(-1.48 \mathrm{~kg}, p<0.0001)$, which accounted for two-thirds of the total weight loss as measured by dual-energy $\mathrm{x}$-ray absorptiometry. In a subset of patients, magnetic resonance imaging (MRI) assessment was performed and it was shown that dapagliflozin was associated with greater reductions in visceral and subcutaneous adipose tissue compared with placebo. However, the hepatic lipid content assessed by MRI did not change significantly with dapagliflozin compared with placebo $(p=0.4499)$ [Bolinder et al. 2012].

In a 78-week site- and patient-blinded extension period of the above trial, which included 140 patients $(76.9 \%)$, dapagliflozin administration resulted in sustained reductions in $\mathrm{HbAlc}$, body weight and fat mass [Bolinder et al. 2014]. Hypoglycemia or meaningful changes in markers of bone turnover or bone mineral density were not observed with dapagliflozin compared with placebo. In this study, the rate of urinary tract and genital infections was similar between dapagliflozin and placebo [Bolinder et al. 2014].

Another 52-week, double-blind trial randomized 814 patients with T2DM receiving metformin monotherapy to add-on dapagliflozin $(n=406)$ or glipizide ( $n=408$, noninferiority trial) [Nauck 
et al. 2011]. Dapagliflozin produced similar reduction in HbA1c compared with glipizide, but induced weight loss in contrast with weight increase with sulphonylurea [Nauck et al. 2011].

\section{Addition to DPP-4 inhibitors}

In a 24 -week, double-blind trial with a 24 -week blinded extension period, 432 patients with T2DM receiving sitagliptin $(100 \mathrm{mg} /$ day $)$ with or without metformin ( $\geqslant 1500 \mathrm{mg} /$ day) were randomized to dapagliflozin $10 \mathrm{mg} /$ day or placebo (Table 1). The administration of dapagliflozin resulted in a significant reduction in HbA1c levels $(-0.5 \%)$ and body weight $(-1.8 \mathrm{~kg})$ compared with placebo, independently of the background treatment [Jabbour et al. 2014]. These results were maintained throughout the extension period. During the trial, patients receiving dapagliflozin experienced signs and symptoms suggestive of genital infection more frequently $(9.8 \%$ versus $0.4 \%)$, but not of urinary tract infection ( $6.7 \%$ versus $6.2 \%)$, compared with placebo [Jabbour et al. 2014].

\section{Addition to pioglitazone}

A study randomized 420 patients with T2DM receiving pioglitazone monotherapy to dapagliflozin 5 or $10 \mathrm{mg} /$ day or placebo for 48 weeks (Table 1) [Rosenstock et al. 2012]. The administration of dapagliflozin for 24 weeks resulted in greater reductions in $\mathrm{HbAlc}(-0.82 \%$ and $-0.97 \%$ with 5 and $10 \mathrm{mg}$, respectively) compared with placebo $(-0.42 \%, p<0.001)$. The reduction in $\mathrm{HbAlc}$ after 48 weeks was $-0.95 \%,-1.21 \%$ and $-0.54 \%$ with dapagliflozin $5 \mathrm{mg}$, dapagliflozin $10 \mathrm{mg}$ or placebo, respectively. Furthermore, the coadministration of dapagliflozin with pioglitazone led to a smaller weight gain at 48 weeks ( $1.35 \mathrm{~kg}$ with $5 \mathrm{mg}$ and $0.69 \mathrm{~kg}$ with $10 \mathrm{mg}$ ) compared with pioglitazone monotherapy $(2.99 \mathrm{~kg})$. Dapagliflozin administration was associated with the occurrence of more events suggestive of genital infection $(9.2 \%$ with $5 \mathrm{mg}$ and $8.6 \%$ with $10 \mathrm{mg}$ ) compared with placebo $(2.9 \%)$, but not with events suggestive of urinary tract infection $(8.5 \%$ and $5.0 \%$ for dapagliflozin 5 or $10 \mathrm{mg}$, respectively, and $7.9 \%$ with placebo). Of interest, the addition of dapagliflozin to pioglitazone resulted in the development of less edema ( $4.3 \%$ with $5 \mathrm{mg}$ and $2.1 \%$ with $10 \mathrm{mg}$ ) compared with pioglitazone/placebo treatment (6.5\%). Two patients (one female and one male) receiving dapagliflozin $5 \mathrm{mg}$ had limb fractures (one foot and one hand fracture, respectively) during the first 24 weeks. One patient receiving dapagliflozin $5 \mathrm{mg}$ was found to have urothelial bladder cancer on day 144. Heart failure was observed in one patient from the placebo group [Rosenstock et al. 2012].

\section{Addition to sulphonylureas}

A 24-week, double-blind trial randomized 597 patients with T2DM receiving glimepiride to dapagliflozin $2.5,5$ or $10 \mathrm{mg} /$ day or placebo (Table 1) [Strojek et al. 2011]. At the end of the trial, the reduction in $\mathrm{HbAlc}$ was $-0.58 \%$, $-0.63 \%,-0.82 \%$ and $-0.13 \%$ with dapagliflozin $2.5,5,10 \mathrm{mg}$ and placebo, respectively (all $p$ $<0.0001$ versus placebo). The administration of dapagliflozin (all doses) led to greater reductions in fasting plasma glucose and body weight. The coadministration of dapagliflozin with glimepiride resulted in more serious adverse events and hypoglycemic events compared with glimepiride/ placebo [Strojek et al. 2011]. Similar improvements in glycemic control and body weight were observed during a 24-week extension period [Strojek et al. 2014].

\section{Addition to insulin}

A 24-week trial randomized 808 patients with inadequately controlled T2DM, despite receiving at least 30 units of insulin daily with or without up to two oral antidiabetic drugs, to dapagliflozin 2.5, 5 or $10 \mathrm{mg}$ or placebo (Table 1) [Wilding et al. 2012]. The administration of dapagliflozin led to greater reductions in $\mathrm{HbA} 1 \mathrm{c}$ compared with placebo (mean difference of $-0.4 \%$ with 2.5 $\mathrm{mg},-0.49 \%$ with $5 \mathrm{mg}$ and $-0.57 \%$ with $10 \mathrm{mg}$ ). Patients receiving dapagliflozin reduced the insulin dose by $0.63-1.95$ units, whereas patients receiving placebo increased their daily dose by 5.65 units. A similar trend was observed with body weight, which decreased by $0.92-1.61 \mathrm{~kg}$ in patients receiving dapagliflozin and increased by $0.43 \mathrm{~kg}$ in those receiving placebo. Similar results were observed during a 24-week extension period. However, patients receiving dapagliflozin experienced a higher rate of hypoglycemic episodes and events suggesting genital or urinary tract infection [Wilding et al. 2012]. These observations were maintained over a total of 104 weeks [Wilding et al. 2014].

Another double-blind trial randomized 71 patients with T2DM to dapagliflozin 10 or $20 \mathrm{mg} /$ day or placebo, in addition to oral antidiabetic drugs and $50 \%$ of their daily insulin dose (initial 
daily dose 84.5-90.0 units) [Wilding et al. 2009]. After 12 weeks, the administration of dapagliflozin led to reductions in $\mathrm{HbAlc}(-0.61 \%$ with $10 \mathrm{mg}$ and $-0.69 \%$ with $20 \mathrm{mg}$ ) compared with placebo (+0.09\%). Furthermore, $65.2 \%$ of patients receiving dapagliflozin experienced a greater than $0.5 \%$ reduction in HbAlc compared with $15.8 \%$ of patients receiving placebo. Body weight decreased more with dapagliflozin $(-4.5$ and $-4.3 \mathrm{~kg}$ with 10 and $20 \mathrm{mg}$, respectively) compared with placebo $(-1.9 \mathrm{~kg})$. A greater percentage of genital infections was observed with dapagliflozin $20 \mathrm{mg}$ compared with placebo [Wilding et al. 2009].

\section{Additional comments}

A meta-analysis of trials examining dapagliflozin in dual or triple therapy showed that dapagliflozin $10 \mathrm{mg}$ reduces $\mathrm{HbA} 1 \mathrm{c}$ by $-0.54 \%$ (weighted mean differences $95 \% \mathrm{CI}-0.40$ to -0.67$)$ and body weight by $-1.81 \mathrm{~kg}$ (weighted mean differences $95 \%$ CI -1.57 to -2.04 ) compared with placebo [Clar et al. 2012]. Another meta-analysis showed that dapagliflozin reduced $\mathrm{HbAlc}$ by $-0.08 \%$ (95\% credible interval -0.25 to 0.10 ) compared with DPP-4 inhibitors, by $-0.02 \%$ $(-0.24$ to 0.21$)$ compared with thiazolidinediones and by $0 \%$ ( -0.16 to 0.16$)$ compared with sulphonylureas [Goring et al. 2014]. Furthermore, dapagliflozin reduced body weight by $-2.74 \mathrm{~kg}$ $(-5.35$ to -0.10$)$ compared with DPP-4 inhibitors and by $-4.67 \mathrm{~kg}(-7.03$ to -2.35$)$ compared with sulphonylureas [Goring et al. 2014]. The reduction in body weight with dapagliflozin is a beneficial action of the drug since it is associated with better glycemic control and improvement of cardiovascular risk factors in patients with T2DM [Filippatos et al. 2005; Filippatos and Elisaf, 2010a; Seifalian et al. 2010].

Dapagliflozin does not induce any major changes in lipid levels, but although not consistently, it has been associated in some trials with a reduction in triglyceride concentration and an increase in high-density lipoprotein cholesterol levels (Table 1). These effects may be associated with an improvement in cardiovascular risk [Gazi et al. 2006; Filippatos and Elisaf, 2013b]. Interestingly, the effects of dapagliflozin on glycemic control and body weight are similar in patients with earlystage and late-stage T2DM [Zhang et al. 2010].

Serum uric acid levels are usually increased in patients with metabolic syndrome and T2DM and are associated through several mechanisms with many other cardiovascular risk factors [Tsouli et al. 2006]. Indeed, serum uric concentration has been shown to be a predictor of cardiovascular disease mortality in healthy middle-aged men [Niskanen et al. 2004]. Dapagliflozin treatment is associated with a reduction in serum uric acid levels, which appears to be dose related (Table 1). The decrease in serum uric acid concentration is possibly attributed to the inhibition of sodium-coupled uric acid reabsorption in the renal proximal tubule [Ferrannini, 2010]. The decrease in uric acid levels represents a beneficial effect of the drug in patients with T2DM, which may be related to a reduction in cardiovascular risk. Furthermore, a possible interrelation has been proposed between increased uric acid levels and nonalcoholic fatty liver disease [Katsiki et al. 2011b]. The presence of nonalcoholic fatty liver is frequent in patients with T2DM, is considered to increase their cardiovascular risk and represents a possible target of therapy [Filippatos and Elisaf, 2010b, 2011; Katsiki et al. 2011a; Ballestri et al. 2014]. Dapagliflozin treatment could be associated with an improvement in nonalcoholic fatty liver disease, since it improves body weight, insulin resistance and triglyceride levels. However, the effects of dapagliflozin in patients with nonalcoholic fatty liver disease have not been systematically assessed in clinical trials and should be a specific target in future studies.

Diabetic nephropathy is a major complication of T2DM. Possible beneficial effects of antidiabetic drugs on kidney histology and function are under investigation [Filippatos and Elisaf, 2013a; Gosmanov et al. 2014]. Dapagliflozin treatment in animal models improves diabetic nephropathy through the improvement of glucose levels and a reduction in inflammation and oxidative stress [De Nicola et al. 2014; Terami et al. 2014]. These effects seem promising for the treatment of diabetic nephropathy, but more studies are needed.

\section{Adverse effects}

Dapagliflozin increases diuresis and modestly decreases blood pressure. Therefore, the drug should be given with caution in patients with high risk of volume depletion, hypotension and electrolyte derangements. Dapagliflozin should not be administrated in patients who are volume depleted, for example those receiving loop diuretics or having acute illness [European Commission, 
Table 1. Selected studies showing the effects of dapagliflozin compared with placebo on anthropometric, glycemic and atherosclerosis-related variables.

Study
Dapagliflozin $1 \mathrm{mg}$ versus
dapagliflozin $2.5 \mathrm{mg}$ versus
dapagliflozin $5 \mathrm{mg}$ versus placebo in
treatment naïve patients with T2DM
( $n=282$, duration 24 weeks) [Bailey
et al. 2012]
Dapagliflozin 2.5 mg versus
dapagliflozin $5 \mathrm{mg}$ versus
dapagliflozin 10 mg versus placebo
in treatment naïve patients with
T2DM (n = 485, duration 24 weeks)
[Ferrannini et al. 2010 ]
Dapagliflozin 5 mg versus
dapagliflozin 10 mg versus placebo
in drug-naïve Asian patients with
T2DM (n=393, duration 24 weeks)
[Ji et al. 2014]

Dapagliflozin $10 \mathrm{mg}$ lone of multiple dosage schemes in this studyl versus metformin $1500 \mathrm{mg} /$ day versus placebo ( $n=389$, duration 12 weeks) [List et al. 2009]

\section{Dapagliflozin 2.5 mg versus}

dapagliflozin $5 \mathrm{mg}$ versus dapagliflozin $10 \mathrm{mg}$ versus placebo on top of metformin ( $n=546$, duration 24 weeks) [Bailey et al. 2010]

Dapagliflozin $2.5 \mathrm{mg}$ versus dapagliflozin $5 \mathrm{mg}$ versus dapagliflozin $10 \mathrm{mg}$ versus placebo on top of metformin $(n=546$, extension for 78 weeks of the above study] [Bailey et al. 2013]

Dapagliflozin $10 \mathrm{mg}$ versus placebo on top of sitagliptin \pm metformin ( $n=432$, duration 24 weeks) [Jabbour et al. 2014]

Dapagliflozin $5 \mathrm{mg}$ versus dapagliflozin $10 \mathrm{mg}$ versus placebo on top of pioglitazone $(n=420$, duration 24 weeks) [Rosenstock et al. 2012]

Dapagliflozin 2.5 mg versus dapagliflozin $5 \mathrm{mg}$ versus dapagliflozin $10 \mathrm{mg}$ versus placebo on top of glimepiride $(n=597$, duration 24 weeks) [Strojek et al. 2011]

\section{Effects*}

$\mathrm{HbA} 1 \mathrm{c}(-0.68$ versus -0.72 versus -0.82 versus $+0.02 \%, p<0.001)$, fasting plasma glucose $(-10.8$ versus -21.6 versus -28.4 versus $+4.1 \mathrm{mg} / \mathrm{dl}, p<0.01)$, body weight $(-2.69$ versus -2.64 versus -2.69 versus $-0.96 \mathrm{~kg}, p<0.01$ l

$\mathrm{HbA} 1 \mathrm{c}(-0.58$ versus -0.77 versus -0.89 versus $-0.23 \%, p<0.001$ for 5 and $10 \mathrm{mg})$, fasting plasma glucose ( -15.2 versus -24.1 versus -28.8 versus $-4.1 \mathrm{mg} / \mathrm{dl}, p<0.001$ for 5 and 10 $\mathrm{mg}$ ), body weight ( -3.3 versus -2.8 versus -3.2 versus $-2.2 \mathrm{~kg}, p=\mathrm{NS}$ )

$\mathrm{HbA} 1 \mathrm{c}(-1.04$ versus -1.11 versus $-0.29 \%, p<0.001)$, fasting plasma glucose $(-25.1$ versus -31.6 versus $+2.5 \mathrm{mg} / \mathrm{dl}, p<0.001$ ), patients with $\mathrm{HbA1c}<7 \%$ at week 24 (42.6 versus 49.8 versus $21.3 \%, p<0.001)$, 2 h postprandial glucose $(-46.8$ versus -56.9 versus $+1.1 \mathrm{mg} / \mathrm{dl}$, $p<0.001)$, body weight $(-1.64$ versus -2.25 versus $-0.27 \mathrm{~kg}, p<0.001)$, patients with $>5 \%$ reduction in body weight (20.8 versus 29.6 versus $5.6 \% ; p<0.05)$, HDL-C $(+9.55$ versus +11.52 versus $+4.24 \%, p$ not mentioned), triglycerides ( -19.11 versus -16.47 versus $-6.95 \%$, $p$ not mentioned), fasting $C$ peptide $(-0.36$ versus -0.40 versus $+0.03 \mathrm{ng} / \mathrm{ml}, p$ not mentioned) $\mathrm{HbA} 1 \mathrm{c}(-0.85$ versus -0.83 versus $-0.18 \%, p<0.001)$, fasting plasma glucose $(-21$ versus -18 versus $-6 \mathrm{mg} / \mathrm{dl}, p=0.002)$, systolic blood pressure $(-6.4$ versus -0.4 versus $+2.4 \mathrm{mmHg}$, $p=0.001)$, diastolic blood pressure $(-2.6$ versus -0.6 versus $+0.3, p=0.07)$, serum creatinine $(-0.02$ versus -0.02 versus $0.0 \mathrm{mg} / \mathrm{dl}, p=0.34)$, blood urea nitrogen $(+2.3$ versus -0.18 versus $-0.96 \mathrm{mg} / \mathrm{dl}, p<0.001)$, serum sodium ( -0.15 versus -0.06 versus $+0.93 \mathrm{mEq} /$ liter, $p=0.05)$, potassium ( -0.0 versus -0.04 versus $-0.01 \mathrm{mEq} / \mathrm{liter}, p=0.88)$, calcium $(-0.12$ versus -0.09 versus $-0.10 \mathrm{mg} / \mathrm{dl}, p=0.88$ ), magnesium ( +0.12 versus -0.3 versus $+0.04 \mathrm{mEq} /$ liter, $p=0.03$ ), phosphate $(+0.12$ versus -0.08 versus $+0.08 \mathrm{mg} / \mathrm{dl}, p=0.73)$, uric acid $(-0.98$ versus +0.18 versus $-0.16 \mathrm{mg} / \mathrm{dl}, p<0.001$ ), hematocrit ( +1.95 versus -1.12 versus $-0.08 \%, p<0.001$ ) $\mathrm{HbA1}$ c $(-0.67$ versus -0.70 versus -0.84 versus $-0.30 \%, p<0.001)$, fasting plasma glucose $(-17.8$ versus -21.4 versus -23.4 versus $-5.9 \mathrm{mg} / \mathrm{dl}, p<0.002)$, patients with $\mathrm{HbA} 1 \mathrm{c}<7.0 \%$ at week 24 ( 33.0 versus 37.5 versus 40.6 versus $25.9 \%, p<0.05$ for 5 and $10 \mathrm{mg}$ ), body weight $(-2.2$ versus -3.0 versus -2.9 versus $-0.9 \mathrm{~kg}, p<0.001)$

$\mathrm{HbA} 1 \mathrm{c}(-0.48$ versus -0.58 versus -0.78 versus $+0.02 \%, p<0.001)$, fasting plasma glucose ( -19.3 versus -26.5 versus -24.5 versus $-10.4 \mathrm{mg} / \mathrm{dl}, p<0.002$ for 5 and $10 \mathrm{mg}$ ), percentage of patients with $\mathrm{HbA} 1 \mathrm{c}<7 \%$ (20.7 versus 26.4 versus 31.5 versus $15.4 \%, p<0.02$ for 5 and $10 \mathrm{mg})$, body weight $(-1.1$ versus -1.7 versus -1.74 versus $+1.36 \mathrm{~kg}, p<0.001)$, uric acid $(-0.6$ versus -0.5 versus -0.6 versus $-0.02 \mathrm{mg} / \mathrm{dl}, p<0.006)$, hematocrit $1+0.84$ versus +1.35 versus +1.84 versus $-1.43 \%, p<0.0001)$, hemoglobin $(+1.5$ versus +3.1 versus +4.1 versus $-4.9 \mathrm{~g} / \mathrm{l}, p<0.0001)$, systolic blood pressure $(+0.7$ versus -1.1 versus -0.3 versus +1.5 $\mathrm{mmHg}, p<0.05$ for 5 and $10 \mathrm{mgl}$

$\mathrm{HbA} 1 \mathrm{c}(-0.5$ versus $0.0 \%, p<0.001), \mathrm{HbA} 1 \mathrm{c}$ in patients with baseline $\mathrm{HbA} 1 \mathrm{c} \geqslant 8 \%$ $(-0.8$ versus $0.0 \%, p<0.001)$, body weight $(-2.1$ versus $-0.3 \mathrm{~kg}, p<0.001)$, fasting plasma glucose $(-24.1$ versus $-3.8 \mathrm{mg} / \mathrm{dl}, p<0.001)$, hematocrit $(+2.2$ versus $-0.5, p$ not mentioned), uric acid ( -0.76 versus $+0.1 \mathrm{mg} / \mathrm{dl}, p$ not mentioned)

$\mathrm{HbA} 1 \mathrm{c}(-0.82$ versus -0.97 versus $-0.42 \%, p<0.001)$, fasting plasma glucose $(-24.9$ versus -29.6 versus $-5.5 \mathrm{mg} / \mathrm{dl}, p<0.001)$, 2 h postprandial glucose $(-65.1$ versus -67.5 versus $-14.1 \mathrm{mg} / \mathrm{dl}, p<0.001)$, body weight ( +0.09 versus -0.14 versus $+1.64 \mathrm{~kg}, p<0.001)$, uric acid $(-0.2$ versus -0.3 versus $+0.1 \mathrm{mg} / \mathrm{dl}, p=\mathrm{NS})$, sodium $(+0.4$ versus +0.7 versus $-0.2 \mathrm{mEq} /$ liter, $p=\mathrm{NS}$ ), parathyroid hormone ( +4.6 versus +4.2 versus $+0.4 \mathrm{pg} / \mathrm{ml}, p=\mathrm{NS}$ )

$\mathrm{HbA} 1 \mathrm{c}(-0.58$ versus -0.63 versus -0.82 versus $-0.13 \%, p<0.001)$, body weight $(-1.18$ versus -1.56 versus -2.26 versus $-0.72 \mathrm{~kg}, p<0.01$ for 5 and $10 \mathrm{mg}$ ), systolic blood pressure $(-4.7$ versus -4.0 versus -5.0 versus $-1.2 \mathrm{mmHg}, p<0.05)$, diastolic blood pressure $(-1.1$ versus -1.7 versus -2.8 versus $-1.4 \mathrm{mmHg} ; p=\mathrm{NS})$, uric acid $(-0.36$ versus -0.44 versus -0.44 versus $+0.02 \mathrm{mg} / \mathrm{dl}$, $p$ not mentioned), triglycerides ( -5.25 versus -3.99 versus -10.56 versus $+0.29 \%, p<0.05$ for $10 \mathrm{mgl}$ 
Table 1. (Continued)

\begin{tabular}{ll}
\hline Study & Effects* \\
\hline $\begin{array}{l}\text { Dapagliflozin } 2.5 \mathrm{mg} \text { versus } \\
\text { dapagliflozin } 5 \mathrm{mg} \text { versus }\end{array}$ & HbA1c (-0.79 versus -0.96 versus -1.01 versus $-0.47 \%, p<0.001)$, body weight $(-1.78$ \\
dapagliflozin $10 \mathrm{mg}$ versus & -0.16 versus $+0.04 \mathrm{mg} / \mathrm{dl}, p$ not mentioned), urinary albumin/creatinine ratio $(-22.1$ versus \\
placebo on top of insulin plus oral & -24.8 versus -17.3 versus $-1.6 \mathrm{mg} / \mathrm{g}, p$ not mentioned), magnesium (+0.16 versus +0.12 \\
antidiabetic drugs ( $n=804$, duration & versus +0.18 versus $-0.12 \mathrm{mEq} / \mathrm{liter}, p$ not mentioned) \\
48 weeks) [Wilding et al. 2012] & \\
\hline${ }^{*} p$ denotes significance versus placebo. & \\
HbA1c, glycosylated hemoglobin; HDL-C, high-density lipoprotein cholesterol; NS, nonsignificant; T2DM, type 2 diabetes mellitus.
\end{tabular}

2012]. Furthermore, a temporal discontinuation of the drug is recommended for patients who develop volume depletion until the depletion is corrected.

Dapagliflozin was associated with more frequent urinary tract infections compared with placebo in clinical studies (Table 2). The percentage of diagnosed urinary tract infections in a recent safety analysis was $3.6 \%$ for dapagliflozin $2.5 \mathrm{mg}(n=$ $814), 5.7 \%$ for dapagliflozin $5 \mathrm{mg}(n=1145)$, $4.3 \%$ for dapagliflozin $10 \mathrm{mg}(n=1193)$ and $3.7 \%$ for placebo $(n=1393)$ [Johnsson et al. 2013a]. Urinary tract infections were observed in a greater proportion in patients with a history of recurrent urinary tract infection (17.1-21.1\%) compared with those without a history of recurrent infection (3.2-5.5\%). Hence, the administration of dapagliflozin should be avoided in patients with a history of recurrent urinary tract infection in order to reduce the incidence of these infections, the related cost of treatment and the disturbance in the quality of life of affected patients. Most of the urinary tract infections responded to one course of standard antimicrobial treatment. The percentage of urinary tract infections which required more than one course of treatment was $5.7 \%, 1.3 \%$ and $15.9 \%$ with dapagliflozin $2.5,5$ and $10 \mathrm{mg}$, respectively, and $14.3 \%$ with placebo. However, the rate of recurrence of urinary tract infections was greater with dapagliflozin (15.3$19.6 \%)$ compared with placebo $(7.7 \%)$. Indeed, in the trials up to 2 years, the rate of recurrent urinary tract infection was $22.9 \%$ with dapagliflozin and $13.6 \%$ with placebo. The possibility of recurrence of urinary tract infection did not appear to be dose dependent and no clear trend for recurrence was observed with dapagliflozin compared with placebo in the group of patients with a history of recurrent urinary tract infection. Pyelonephritis was observed at a low rate, similar to placebo [Johnsson et al. 2013a].
An increased rate of genital infections was observed with dapagliflozin in clinical studies (Table 2). A safety analysis showed that the percentage of vulvovaginitis or balanitis was $4.1 \%$ for dapagliflozin $2.5 \mathrm{mg}(n=814), 5.7 \%$ for dapagliflozin $5 \mathrm{mg}(n=1145), 4.8 \%$ for dapagliflozin 10 $\operatorname{mg}(n=1193)$ and $0.9 \%$ for placebo $(n=1393)$ [Johnsson et al. 2013b]. Genital infection was observed in a greater percentage of women than men. The more frequent genital infections in women treated with dapagliflozin were vaginal infection, vulvovaginal mycotic infection, vulvovaginal candidiasis and genital fungal infection, whereas balanitis was the most frequent genital infection in men receiving dapagliflozin. In patients without a history of recurrent genital yeast infection, a small increase in genital infections was observed with dapagliflozin (3.9-5.9\%) compared with placebo $(0.8 \%)$. However, in the small number of patients with a history of recurrent genital yeast infection, genital infections were reported in a greater percentage of those receiving dapagliflozin (23-50\%) compared with placebo $(10 \%)$. Based on these observations, the administration of dapagliflozin should be avoided in patients with a history of recurrent genital yeast infection. Genital infections were usually mild to moderate and treated with standard antimicrobial medication. Recurrence of genital infections was reported more frequently in patients receiving dapagliflozin $(18.1 \%)$ compared with placebo $(8.3 \%)$. In studies up to 2 years, recurrence of genital infections was also more frequent with dapagliflozin compared with placebo (27.2 versus $22.2 \%$, respectively) [Johnsson et al. 2013b].

Dapagliflozin has been shown to be cost effective when added to insulin and it is not associated with a reduction in health-related quality of life [Grandy et al. 2014a, 2014b; Van Haalen et al. 2014]. However, it is obvious that the cost of treatment will increase and the quality of life will 
Table 2. Adverse effects with increased incidence compared with placebo in selected studies of dapagliflozin.

Study design
Dapagliflozin $1 \mathrm{mg}$ versus dapagliflozin
$2.5 \mathrm{mg}$ versus dapagliflozin $5 \mathrm{mg}$ versus
placebo in treatment naïve patients
with T2DM ( $n=282$, duration 24 weeks]
[Bailey et al. 2012]
Dapagliflozin $2.5 \mathrm{mg}$ versus
dapagliflozin 5 mg versus dapagliflozin
10 mg versus placebo in treatment naïve
patients with T2DM ( $n=485$, duration 24
weeks) [Ferrannini et al. 2010]
Dapagliflozin 5 mg versus dapagliflozin
10 mg versus placebo in drug-naïve
Asian patients with T2DM ( $n=393$,
duration 24 weeks) [Ji et al. 2014]

Dapagliflozin $10 \mathrm{mg}$ lone of multiple dosage schemes in this study) versus metformin $1500 \mathrm{mg} /$ day versus placebo ( $n=389$, duration 12 weeks) [List et al. 2009]

Dapagliflozin $2.5 \mathrm{mg}$ versus dapagliflozin $5 \mathrm{mg}$ versus dapagliflozin $10 \mathrm{mg}$ versus placebo on top of metformin ( $n=546$, duration 24 weeks) [Bailey et al. 2010]

\section{Dapagliflozin $2.5 \mathrm{mg}$ versus} dapagliflozin $5 \mathrm{mg}$ versus dapagliflozin $10 \mathrm{mg}$ versus placebo on top of metformin ( $n=546$, extension for 78 weeks of the above study] [Bailey et al. 2013]

Dapagliflozin $10 \mathrm{mg}$ versus placebo on top of sitagliptin \pm metformin $(n=432$, duration 48 weeks) [Jabbour et al. 2014] Dapagliflozin $5 \mathrm{mg}$ versus dapagliflozin $10 \mathrm{mg}$ versus placebo on top of pioglitazone $(n=420$, duration 48 weeks] [Rosenstock et al. 2012]

Adverse effects $(\%)$ with increased proportion compared with placebo

At least one drug-related adverse event (6.9 versus 12.2 versus 7.4 versus 11.8 ), nasopharyngitis (6.9 versus 1.4 versus 2.9 versus 4.4), hypoglycemia (0 versus 1.4 versus 1.5 versus 0 ), urinary tract infection (4.2 versus 1.4 versus 2.9 versus 1.5 ), genital infections (1.4 versus 6.8 versus 2.9 versus 2.9 ), hypotension/dehydration/ hypovolemia (0 versus 0 versus 1.5 versus 0 )

At least one adverse event ( 63.1 versus 57.8 versus 68.6 versus 60 ), discontinuation due to adverse events (3.1 versus 4.7 versus 7.1 versus 1.3), events suggestive of urinary tract infection (4.6 versus 12.5 versus 5.7 versus 4), events suggestive of genital infection (7.7 versus 7.8 versus 12.9 versus 1.3), nasopharyngitis (10.8 versus 4.7 versus 2.9 versus 5.3 ), diarrhea (6.2 versus 1.6 versus 1.4 versus 1.3 )

Nasopharyngitis (5.5 versus 3.0 versus 3.8), urinary tract infection (3.9 versus 3.8 versus 3.0 ), toothache (3.1 versus 2.3 versus 1.5), diarrhea (2.3 versus 2.3 versus 1.5 ), thrombocytopenia (3.9 versus 2.3 versus 0 ), back pain (0.8 versus 3.0 versus 0.8 ), constipation (1.6 versus 2.3 versus 0.8 ), gastritis ( 2.3 versus 0.8 versus 0 ), renal impairment ( 0.8 versus 2.3 versus 1.5), genital infection ( 3.1 versus 4.5 versus 0.8 ) Total subjects with an adverse event ( 68 versus 68 versus 54 ), serious adverse events ( 2 versus 2 versus 0), discontinuation for adverse events (6 versus 2 versus 2), urinary tract or genital infection (10.6 versus 10.7 versus 5.6 ), diarrhea (2 versus 13 versus 7), headache ( 4 versus 4 versus 11), nausea ( 6 versus 11 versus 6), hypoglycemic events (6 versus 9 versus 41

One or more drug-related adverse event ( 16 versus 18 versus 23 versus 16), headache ( 3 versus 7 versus 8 versus 4), back pain ( 4 versus 2 versus 7 versus 5), diarrhea ( 2 versus 4 versus 7 versus 5), urinary tract infection (3 versus 5 versus 7 versus 5), influenza (9 versus 9 versus 6 versus 7), nasopharyngitis (9 versus 3 versus 6 versus 8), hypoglycemia ( 2 versus 4 versus 4 versus 3), events suggestive of genital infection ( 8 versus 13 versus 9 versus 5 )

At least one related adverse event ( 26.3 versus 24.1 versus 33.1 versus 20.4 ), back pain ( 8 versus 5.3 versus 13.3 versus 8), influenza (13.9 versus 14.1 versus 12.6 versus 10.9), diarrhea (5.1 versus 6.6 versus 11.9 versus 7.3), headache (7.3 versus 9.5 versus 11.1 versus 5.8 ), nasopharyngitis (11.7 versus 5.8 versus 8.9 versus 8.8 ), events suggestive of urinary tract infection ( 8.0 versus 8.8 versus 13.3 versus 8.0 ), events suggestive of genital infection (11.7 versus 14.6 versus 12.6 versus 5.1 )

At least one adverse event (66.2 versus 61.1), events suggestive of genital infection (9.8 versus 0.4 ), events suggestive of urinary tract infection (6.7 versus 6.2 ), diagnosed events of urinary tract infection (5.8 versus 3.5)

At least one adverse event (68.1 versus 70.7 versus 66.9), at least one serious adverse event ( 4.3 versus 1.4 versus 2.9), nasopharyngitis (5.0 versus 7.9 versus 5.0 ), diarrhea (3.5 versus 6.4 versus 4.3), back pain (3.5 versus 5.7 versus 2.9), pain in extremity (7.1 versus 2.9 versus 0.7 ), events suggestive of urinary tract infection ( 8.5 versus 5.0 versus 7.9 ), events suggestive of genital infection (9.2 versus 8.6 versus 2.9 ), mycotic genital infection (5.7 versus 4.3 versus 0.7 ), decreased renal function (1.4 versus 1.4 versus 0.7)

Dapagliflozin $2.5 \mathrm{mg}$ versus dapagliflozin $5 \mathrm{mg}$ versus dapagliflozin $10 \mathrm{mg}$ versus placebo on top of insulin plus oral antidiabetic drugs $(n=804$, duration 48 weeks) [Wilding et al. 2012]

At least one drug-related adverse event (21.3 versus 29.2 versus 29.1 versus 20.8), nasopharyngitis (15.8 versus 16.5 versus 12.8 versus 11.7), urinary tract infection ( 5.4 versus 7.5 versus 7.1 versus 4.1), diarrhea (2.5 versus 5.2 versus 5.1 versus 4.1), constipation (5.9 versus 3.9 versus 3.1 versus 1.5), events suggesting genital infections (6.4 versus 9.9 versus 10.7 versus 2.5$)$, hypoglycemia ( 60.4 versus 55.7 versus 53.6 versus 51.8), major hypoglycemia (1.5 versus 0.9 versus 1.5 versus 1.0 )

T2DM, type 2 diabetes mellitus.

be adversely affected in patients who experience urinary tract or genital infections, especially if those are recurrent. Furthermore, an increase in the use of antibiotics is expected in patients with recurrent infections, which is an important problem in the era of antibiotic overuse and resistance. The effects of dapagliflozin treatment on those variables should be better assessed in dapagliflozin-treated patients with recurrent urinary tract or genital infections. 
Dapagliflozin does not cause hypoglycemia. However, there is an increased risk of hypoglycemia when the drug is given with insulin or sulphonylureas. A decrease in the dose of insulin or sulphonylureas may be required in most patients to reduce the risk of hypoglycemia.

The administration of dapagliflozin is associated with a small increase of hematocrit (1.5-3.0\%). This increase seems dose related and is possibly attributed to hemoconcentration because of osmotic diuresis [Shah et al. 2012]. Dapagliflozin has also been associated with a small increase in serum magnesium, but its concentration generally remained within normal physiological ranges. No changes were observed in serum sodium, potassium or calcium concentration [Tahrani and Barnett, 2010; Whaley et al. 2012].

Concerns have been expressed regarding a possible association of dapagliflozin with neoplasms. An increased incidence of bladder and breast cancer was noted with dapagliflozin compared with control [Vasilakou et al. 2013]. On 19 January 2012 the US Food and Drug administration (FDA) did not approve the use of dapagliflozin due to concerns of increased risk of breast and bladder cancers, and requested additional clinical data [Burki 2012; US FDA, 2012]. This decision was based on the findings of previous studies, in which nine cases of bladder cancer were identified out of 4310 individuals receiving dapagliflozin, whereas one case was identified out of 1962 subjects in the control group. Furthermore, nine cases of breast cancer were detected in patients receiving dapagliflozin and none in the control group [FDA Advisory Committee Meeting, 2011]. It should be mentioned that many patients who were diagnosed with bladder cancer in dapagliflozin trials had preexisting hematuria, a fact that raises the possibility that bladder cancer was already evident. However, it has been speculated that elevated levels of glucose in the bladder owing to the mechanism of action of dapagliflozin may increase the rate of growth of preexisting cancers [Burki, 2012]. Further evidence of animal studies with dapagliflozin showed no evidence that the drug is associated with increased risk of tumor development [Reilly et al. 2014]. Finally, on 8 January 2014, FDA approved the use of dapagliflozin, requiring more trials specifically assessing the risk of bladder cancer development [US FDA, 2014]. However, as a precautionary measure, dapagliflozin is not recommended to be given concomitantly with pioglitazone, because of the small increased risk of bladder cancer with the latter [European Commission, 2012]. Another precautionary measure could be the avoidance of dapagliflozin administration in patients with preexisting undiagnosed hematuria.

A 50-week administration of dapagliflozin in 150 male and postmenopausal female patients with T2DM on metformin treatment did not alter the markers of bone formation and resorption or bone mineral density [Ljunggren et al. 2012]. Dapagliflozin, at supratherapeutic doses, does not affect the QT interval in a clinically meaningful manner in healthy subjects [Carlson et al. 2011].

\section{Drug interactions}

The pharmacokinetics of dapagliflozin are at least partly determined by the fact that it is a substrate but not a significant inhibitor of $\mathrm{P}$ glycoprotein, which is a cell-membrane-associated protein that transports a variety of drug substrates in the intestine, central nervous system, leukocytes, liver and kidney [Matheny et al. 2001]. Dapagliflozin is not an inducer or inhibitor of human P450 enzymes [Obermeier et al. 2010].

The coadministration of dapagliflozin with voglibose, pioglitazone, metformin, glimepiride, sitagliptin, warfarin, digoxin or valsartan does not significantly affect the pharmacokinetic variables of either agent and no dose adjustment is required [Kasichayanula et al. 2011b, 2012; Imamura et al. 2013]. The coadministration with simvastatin did not induce any clinically meaningful effect on the pharmacokinetics of dapagliflozin, but increased the area under the curve for simvastatin by $19 \%$ and simvastatin acid by $30 \%$ [Kasichayanula et al. 2012]. These increases do not seem clinically meaningful, but caution should be taken when dapagliflozin and simvastatin are coadministered. The coadministration of dapagliflozin with rifampin and mefenamic acid resulted in modest changes in dapagliflozin exposure and minor changes in urinary glucose excretion, but these alterations are not considered clinically meaningful [Kasichayanula et al. 2013a].

\section{Conclusion}

Dapagliflozin is an antidiabetic drug, which decreases renal glucose reabsorption by the selective inhibition of the renal SGLT2. Additional actions of dapagliflozin include body weight and 
blood pressure reduction, which could be beneficial in patients with T2DM patients. Dapagliflozin is a useful drug, which can be given as a monotherapy in patients who cannot tolerate metformin or in combination with other antidiabetic drugs (except pioglitazone). Dapagliflozin could be beneficial in overweight or obese patients with T2DM with cardiovascular risk factors, such as hypertension or hyperuricemia. The drug should not be administrated to patients with moderate or severe renal impairment or to patients at risk for developing volume depletion. The drug is associated with increased incidence of genital and lower urinary tract infection, but these infections are usually mild to moderate and respond to standard antimicrobial treatment.

The effects of dapagliflozin on hard cardiovascular endpoints have not been studied. The DECLARE study (Dapagliflozin Effect on Cardiovascular Events A Multicenter, Randomized, Double-Blind, Placebo-Controlled Trial to Evaluate the Effect of Dapagliflozin 10 mg Once Daily on the Incidence of Cardiovascular Death, Myocardial Infarction or Ischemic Stroke in Patients With Type 2 Diabetes) [ClinicalTrials. gov identifier: NCT01730534], a large, randomized, placebo-controlled trial involving more than 17,000 adult patients with T2DM, will examine if dapagliflozin when added to current antidiabetic therapy is effective in reducing cardiovascular events compared with placebo. This study is expected to be completed in 2019 and will provide data regarding the long-term effects and safety profile of dapagliflozin.

\section{Funding}

This review received no specific grant from any funding agency in the public, commercial, or notfor-profit sectors.

\section{Conflict of interest statement}

This review was conducted independently; no company or institution supported it financially. The authors have given talks, attended conferences and participated in trials and advisory boards sponsored by various pharmaceutical companies.

\section{References}

Agouridis, A., Rizos, C., Elisaf, M. and Filippatos, T. (2013) Does combination therapy with statins and fibrates prevent cardiovascular disease in diabetic patients with atherogenic mixed dyslipidemia? Rev Diabet Stud 10: 171-190.

American Diabetes Association (2014) Standards of medical care in diabetes. Diabetes Care 37(Suppl. 1): S14-S80.

Bailey, C., Gross, J., Hennicken, D., Iqbal, N., Mansfield, T. and List, J. (2013) Dapagliflozin add-on to metformin in type 2 diabetes inadequately controlled with metformin: a randomized, doubleblind, placebo-controlled 102-week trial. BMC Med 11: 43 .

Bailey, C., Gross, J., Pieters, A., Bastien, A. and List, J. (2010) Effect of dapagliflozin in patients with type 2 diabetes who have inadequate glycaemic control with metformin: a randomised, double-blind, placebocontrolled trial. Lancet 375: 2223-2233.

Bailey, C., Iqbal, N., T'joen, C. and List, J. (2012) Dapagliflozin monotherapy in drug-naive patients with diabetes: a randomized-controlled trial of lowdose range. Diabetes Obes Metab 14: 951-959.

Balakumar, P., Sundram, K. and Dhanaraj, S. (2014) Dapagliflozin: glucuretic action and beyond. Pharmacol Res 82: 34-39.

Ballestri, S., Lonardo, A., Bonapace, S., Byrne, C., Loria, P. and Targher, G. (2014) Risk of cardiovascular, cardiac and arrhythmic complications in patients with non-alcoholic fatty liver disease. World f Gastroenterol 20: 1724-1745.

Bolinder, J., Ljunggren, O., Johansson, L., Wilding, J., Langkilde, A., Sjostrom, C. et al. (2014) Dapagliflozin maintains glycaemic control while reducing weight and body fat mass over 2 years in patients with type 2 diabetes mellitus inadequately controlled on metformin. Diabetes Obes Metab 16: 159-169.

Bolinder, J., Ljunggren, O., Kullberg, J., Johansson, L., Wilding, J., Langkilde, A. et al. (2012) Effects of dapagliflozin on body weight, total fat mass, and regional adipose tissue distribution in patients with type 2 diabetes mellitus with inadequate glycemic control on metformin. $\mathcal{F}$ Clin Endocrinol Metab 97: 1020-1031.

Boulton, D., Kasichayanula, S., Keung, C., Arnold, M., Christopher, L., Xu, X. et al. (2013) Simultaneous oral therapeutic and intravenous (1) (4)C-microdoses to determine the absolute oral bioavailability of saxagliptin and dapagliflozin. $\mathrm{Br} \mathcal{F}$ Clin Pharmacol 75: 763-768.

Burki, T. (2012) FDA rejects novel diabetes drug over safety fears. Lancet 379: 507.

Carlson, G., Tou, C., Parikh, S., Birmingham, B. and Butler, K. (2011) Evaluation of the effect of dapagliflozin on cardiac repolarization: a thorough QT/QTc study. Diabetes Ther 2: 123-132. 
Clar, C., Gill, J., Court, R. and Waugh, N. (2012) Systematic review of SGLT2 receptor inhibitors in dual or triple therapy in type 2 diabetes. BMF Open 2: e001007.

De Nicola, L., Gabbai, F., Liberti, M., Sagliocca, A., Conte, G. and Minutolo, R. (2014) Sodium/glucose cotransporter 2 inhibitors and prevention of diabetic nephropathy: targeting the renal tubule in diabetes. Am F Kidney Dis 64: 16-24.

European Commission (2012) Dapagliflozin summary of product characteristics. Available at: http://ec.europa.eu/health/documents/communityregister/2012/20121112124487/anx_124487_en.pdf (accessed 28 July 2014).

FDA Advisory Committee Meeting (2011) FDA briefing document. NDA 202293. Available at: www.fda.gov/downloads/AdvisoryCommittees/ CommitteesMeetingMaterials/Drugs/ EndocrinologicandMetabolicDrugsAdvisory Committee/UCM262994.pdf (accessed 2 August 2014).

Ferrannini, E. (2010) Sodium-glucose transporter-2 inhibition as an antidiabetic therapy. Nephrol Dial Transplant 25: 2041-2043.

Ferrannini, E., Ramos, S., Salsali, A., Tang, W. and List, J. (2010) Dapagliflozin monotherapy in type 2 diabetic patients with inadequate glycemic control by diet and exercise: a randomized, double-blind, placebocontrolled, phase 3 trial. Diabetes Care 33: 2217-2224.

Filippatos, T., Athyros, V. and Elisaf, M. (2014) The pharmacokinetic considerations and adverse effects of DPP-4 inhibitors. Expert Opin Drug Metab Toxicol 10: 787-812.

Filippatos, T. and Elisaf, M. (2010a) Combination drug treatment in obese diabetic patients. World $\mathcal{F}$ Diabetes 1: 8-11.

Filippatos, T. and Elisaf, M. (2010b) Combination drug treatment in patients with non-alcoholic fatty liver disease. World f Hepatol 2: 139-142.

Filippatos, T. and Elisaf, M. (2011) Role of ezetimibe in non-alcoholic fatty liver disease. World $\mathcal{f}$ Hepatol 3: 265-267.

Filippatos, T. and Elisaf, M. (2013a) Effects of glucagon-like peptide-1 receptor agonists on renal function. World F Diabetes 4: 190-201.

Filippatos, T. and Elisaf, M. (2013b) High density lipoprotein and cardiovascular diseases. World $\mathcal{F}$ Cardiol 5: 210-214.

Filippatos, T., Kiortsis, D., Liberopoulos, E., Georgoula, M., Mikhailidis, D. and Elisaf, M. (2005) Effect of orlistat, micronised fenofibrate and their combination on metabolic parameters in overweight and obese patients with the metabolic syndrome: the FenOrli study. Curr Med Res Opin 21: 1997-2006.
Gazi, I., Filippatos, T., Tsimihodimos, V., Saougos, V., Liberopoulos, E., Mikhailidis, D. et al. (2006) The hypertriglyceridemic waist phenotype is a predictor of elevated levels of small, dense LDL cholesterol. Lipids 41: 647-654.

Goring, S., Hawkins, N., Wygant, G., Roudaut, M., Townsend, R., Wood, I. et al. (2014) Dapagliflozin compared with other oral anti-diabetes treatments when added to metformin monotherapy: a systematic review and network meta-analysis. Diabetes Obes Metab 16: 433-442.

Gosmanov, A., Wall, B. and Gosmanova, E. (2014) Diagnosis and treatment of diabetic kidney disease. Am F Med Sci 347: 406-413.

Grandy, S., Hashemi, M., Langkilde, A., Parikh, S. and Sjostrom, C. (2014a) Changes in weight lossrelated quality of life among type 2 diabetes mellitus patients treated with dapagliflozin. Diabetes Obes Metab 16: 645-650.

Grandy, S., Langkilde, A., Sugg, J., Parikh, S. and Sjostrom, C. (2014b) Health-related quality of life (EQ-5D) among type 2 diabetes mellitus patients treated with dapagliflozin over 2 years. Int $\mathcal{F}$ Clin Pract 68: 486-494.

Imamura, A., Kusunoki, M., Ueda, S., Hayashi, N. and Imai, Y. (2013) Impact of voglibose on the pharmacokinetics of dapagliflozin in Japanese patients with type 2 diabetes. Diabetes Ther 4: 41-49.

Jabbour, S., Hardy, E., Sugg, J. and Parikh, S.; Study 10 Group (2014) Dapagliflozin is effective as add-on therapy to sitagliptin with or without metformin: a 24-week, multicenter, randomized, double-blind, placebo-controlled study. Diabetes Care 37: 740-750.

Ji, L., Ma, J., Li, H., Mansfield, T., T'joen, C., Iqbal, N. et al. (2014) Dapagliflozin as monotherapy in drugnaive Asian patients with type 2 diabetes mellitus: a randomized, blinded, prospective phase III study. Clin Ther 36: 84-100 e109.

Johnsson, K., Ptaszynska, A., Schmitz, B., Sugg, J., Parikh, S. and List, J. (2013a) Urinary tract infections in patients with diabetes treated with dapagliflozin. $\mathcal{F}$ Diabetes Complications 27: 473-478.

Johnsson, K., Ptaszynska, A., Schmitz, B., Sugg, J., Parikh, S. and List, J. (2013b) Vulvovaginitis and balanitis in patients with diabetes treated with dapagliflozin. F Diabetes Complications 27: 479-484.

Kasichayanula, S., Chang, M., Hasegawa, M., Liu, X., Yamahira, N., Lacreta, F. et al. (2011a) Pharmacokinetics and pharmacodynamics of dapagliflozin, a novel selective inhibitor of sodiumglucose co-transporter type 2, in Japanese subjects without and with type 2 diabetes mellitus. Diabetes Obes Metab 13: 357-365. 
Kasichayanula, S., Chang, M., Liu, X., Shyu, W., Griffen, S., Lacreta, F. et al. (2012) Lack of pharmacokinetic interactions between dapagliflozin and simvastatin, valsartan, warfarin, or digoxin. $A d v$ Ther 29: 163-177.

Kasichayanula, S., Liu, X., Griffen, S., Lacreta, F. and Boulton, D. (2013a) Effects of rifampin and mefenamic acid on the pharmacokinetics and pharmacodynamics of dapagliflozin. Diabetes Obes Metab 15: 280-283.

Kasichayanula, S., Liu, X., Lacreta, F., Griffen, S. and Boulton, D. (2014) Clinical pharmacokinetics and pharmacodynamics of dapagliflozin, a selective inhibitor of sodium-glucose co-transporter type 2. Clin Pharmacokinet 53: 17-27.

Kasichayanula, S., Liu, X., Pe Benito, M., Yao, M., Pfister, M., Lacreta, F. et al. (2013b) The influence of kidney function on dapagliflozin exposure, metabolism and pharmacodynamics in healthy subjects and in patients with type 2 diabetes mellitus. Br f Clin Pharmacol 76: 432-444.

Kasichayanula, S., Liu, X., Shyu, W., Zhang, W., Pfister, M., Griffen, S. et al. (2011b) Lack of pharmacokinetic interaction between dapagliflozin, a novel sodium-glucose transporter 2 inhibitor, and metformin, pioglitazone, glimepiride or sitagliptin in healthy subjects. Diabetes Obes Metab 13: 47-54.

Kasichayanula, S., Liu, X., Zhang, W., Pfister, M., Lacreta, F. and Boulton, D. (2011c) Influence of hepatic impairment on the pharmacokinetics and safety profile of dapagliflozin: an open-label, parallelgroup, single-dose study. Clin Ther 33: 1798-1808.

Kasichayanula, S., Liu, X., Zhang, W., Pfister, M., Reele, S., Aubry, A. et al. (2011d) Effect of a highfat meal on the pharmacokinetics of dapagliflozin, a selective SGLT2 inhibitor, in healthy subjects. Diabetes Obes Metab 13: 770-773.

Katsiki, N., Athyros, V., Karagiannis, A. and Mikhailidis, D. (2011a) Current treatment for nonalcoholic fatty liver disease. Expert Opin Pharmacother 12: 2141-2142.

Katsiki, N., Athyros, V., Karagiannis, A. and Mikhailidis, D. (2011b) Hyperuricaemia and nonalcoholic fatty liver disease (NAFLD): a relationship with implications for vascular risk? Curr Vasc Pharmacol 9: 698-705.

Komoroski, B., Vachharajani, N., Boulton, D., Kornhauser, D., Geraldes, M., Li, L. et al. (2009) Dapagliflozin, a novel SGLT2 inhibitor, induces dose-dependent glucosuria in healthy subjects. Clin Pharmacol Ther 85: 520-526.

Lambers Heerspink, H., De Zeeuw, D., Wie, L., Leslie, B. and List, J. (2013) Dapagliflozin a glucoseregulating drug with diuretic properties in subjects with type 2 diabetes. Diabetes Obes Metab 15: 853-862.
Leong, A., Dasgupta, K., Chiasson, J. and Rahme, E. (2013) Estimating the population prevalence of diagnosed and undiagnosed diabetes. Diabetes Care 36: 3002-3008.

List, J., Woo, V., Morales, E., Tang, W. and Fiedorek, F. (2009) Sodium-glucose cotransport inhibition with dapagliflozin in type 2 diabetes. Diabetes Care 32: 650-657.

Ljunggren, O., Bolinder, J., Johansson, L., Wilding, J., Langkilde, A., Sjostrom, C. et al. (2012) Dapagliflozin has no effect on markers of bone formation and resorption or bone mineral density in patients with inadequately controlled type 2 diabetes mellitus on metformin. Diabetes Obes Metab 14: 990-999.

Matheny, C., Lamb, M., Brouwer, K. and Pollack, G. (2001) Pharmacokinetic and pharmacodynamic implications of P-glycoprotein modulation.

Pharmacotherapy 21: 778-796.

Merovci, A., Solis-Herrera, C., Daniele, G., Eldor, R., Fiorentino, T., Tripathy, D. et al. (2014) Dapagliflozin improves muscle insulin sensitivity but enhances endogenous glucose production. $\mathcal{F}$ Clin Invest 124: 509-514.

Mudaliar, S., Henry, R., Boden, G., Smith, S., Chalamandaris, A., Duchesne, D. et al. (2014) Changes in insulin sensitivity and insulin secretion with the sodium glucose cotransporter 2 inhibitor dapagliflozin. Diabetes Technol Ther 16: 137-144.

Nauck, M., Del Prato, S., Meier, J., Duran-Garcia, S., Rohwedder, K., Elze, M. et al. (2011) Dapagliflozin versus glipizide as add-on therapy in patients with type 2 diabetes who have inadequate glycemic control with metformin: a randomized, 52-week, double-blind, active-controlled noninferiority trial. Diabetes Care 34: 2015-2022.

Niskanen, L., Laaksonen, D., Nyyssonen, K., Alfthan, G., Lakka, H., Lakka, T. et al. (2004) Uric acid level as a risk factor for cardiovascular and all-cause mortality in middle-aged men: a prospective cohort study. Arch Intern Med 164: 1546-1551.

Obermeier, M., Yao, M., Khanna, A., Koplowitz, B., Zhu, M., Li, W. et al. (2010) In vitro characterization and pharmacokinetics of dapagliflozin (BMS512148), a potent sodium-glucose cotransporter type II inhibitor, in animals and humans. Drug Metab Dispos 38: 405-414.

Oliva, R. and Bakris, G. (2014) Blood pressure effects of sodium-glucose co-transport 2 (SGLT2) inhibitors. f Am Soc Hypertens 8: 330-339.

Reilly, T., Graziano, M., Janovitz, E., Dorr, T., Fairchild, C., Lee, F. et al. (2014) Carcinogenicity risk assessment supports the chronic safety of dapagliflozin, an inhibitor of sodium-glucose co-transporter 2 , in the treatment of type 2 diabetes mellitus. Diabetes Ther 5: 73-96. 
Rosenstock, J., Vico, M., Wei, L., Salsali, A. and List, J. (2012) Effects of dapagliflozin, an SGLT2 inhibitor, on $\mathrm{HbA}(1 \mathrm{c})$, body weight, and hypoglycemia risk in patients with type 2 diabetes inadequately controlled on pioglitazone monotherapy. Diabetes Care 35: 1473-1478.

Seifalian, A., Filippatos, T., Joshi, J. and Mikhailidis, D. (2010) Obesity and arterial compliance alterations. Curr Vasc Pharmacol 8: 155-168.

Shah, N., Deeb, W., Choksi, R. and Epstein, B. (2012) Dapagliflozin: a novel sodium-glucose cotransporter type 2 inhibitor for the treatment of type 2 diabetes mellitus. Pharmacotherapy 32: 80-94.

Sheikh-Ali, M., Raheja, P. and Borja-Hart, N. (2013) Medical management and strategies to prevent coronary artery disease in patients with type 2 diabetes mellitus. Postgrad Med 125: 17-33.

Strojek, K., Yoon, K., Hruba, V., Elze, M., Langkilde, A. and Parikh, S. (2011) Effect of dapagliflozin in patients with type 2 diabetes who have inadequate glycaemic control with glimepiride: a randomized, 24-week, double-blind, placebocontrolled trial. Diabetes Obes Metab 13: 928-938.

Strojek, K., Yoon, K., Hruba, V., Sugg, J., Langkilde, A. and Parikh, S. (2014) Dapagliflozin added to glimepiride in patients with type 2 diabetes mellitus sustains glycemic control and weight loss over 48 weeks: a randomized, double-blind, parallel-group, placebo-controlled trial. Diabetes Ther 5: 267-283.

Tahrani, A. and Barnett, A. (2010) Dapagliflozin: a sodium glucose cotransporter 2 inhibitor in development for type 2 diabetes. Diabetes Ther 1 : 45-56.

Terami, N., Ogawa, D., Tachibana, H., Hatanaka, T., Wada, J., Nakatsuka, A. et al. (2014) Long-term treatment with the sodium glucose cotransporter 2 inhibitor, dapagliflozin, ameliorates glucose homeostasis and diabetic nephropathy in $\mathrm{db} / \mathrm{db}$ mice. PLoS One 9: e100777.

Tsouli, S., Liberopoulos, E., Mikhailidis, D., Athyros, V. and Elisaf, M. (2006) Elevated serum uric acid levels in metabolic syndrome: an active component or an innocent bystander? Metabolism 55: 1293-1301.

US FDA (2012) FDA declined dapagliflozin. US Food and Drug Administration. Available at: http:// www.medscape.com/viewarticle/757175 (accessed 1 August 2014).

US FDA (2014) FDA approves dapagliflozin. US Food and Drug Administration. Available at: http://www.fda.gov/NewsEvents/Newsroom/ PressAnnouncements/ucm380829.htm (accessed 1 August 2014).

Ussher, J., Sutendra, G. and Jaswal, J. (2012) The impact of current and novel anti-diabetic therapies on cardiovascular risk. Future Cardiol 8: 895-912.

Vallon, V., Platt, K., Cunard, R., Schroth, J., Whaley, J., Thomson, S. et al. (2011) SGLT2 mediates glucose reabsorption in the early proximal tubule. $\mathcal{F}$ Am Soc Nephrol 22: 104-112.

Van Haalen, H., Pompen, M., Bergenheim, K., Mcewan, P., Townsend, R. and Roudaut, $M$. (2014) Cost effectiveness of adding dapagliflozin to insulin for the treatment of type 2 diabetes mellitus in the Netherlands. Clin Drug Investig 34: 135-146.

Vasilakou, D., Karagiannis, T., Athanasiadou, E., Mainou, M., Liakos, A., Bekiari, E. et al. (2013) Sodium-glucose cotransporter 2 inhibitors for type 2 diabetes: a systematic review and meta-analysis. Ann Intern Med 159: 262-274.

Whaley, J., Tirmenstein, M., Reilly, T., Poucher, S., Saye, J., Parikh, S. et al. (2012) Targeting the kidney and glucose excretion with dapagliflozin: preclinical and clinical evidence for SGLT2 inhibition as a new option for treatment of type 2 diabetes mellitus. Diabetes Metab Syndr Obes 5: 135-148.

Wilding, J., Norwood, P., T'joen, C., Bastien, A., List, J. and Fiedorek, F. (2009) A study of dapagliflozin in patients with type 2 diabetes receiving high doses of insulin plus insulin sensitizers: applicability of a novel insulin-independent treatment. Diabetes Care 32: 1656-1662.

Wilding, J., Woo, V., Rohwedder, K., Sugg, J. and Parikh, S.; Dapaglifozin 006 Study Group (2014) Dapagliflozin in patients with type 2 diabetes receiving high doses of insulin: efficacy and safety over 2 years. Diabetes Obes Metab 16: 124-136.

Wilding, J., Woo, V., Soler, N., Pahor, A., Sugg, J., Rohwedder, K. et al. (2012) Long-term efficacy of dapagliflozin in patients with type 2 diabetes mellitus receiving high doses of insulin: a randomized trial. Ann Intern Med 156: 405-415.

Zhang, L., Feng, Y., List, J., Kasichayanula, S. and Pfister, M. (2010) Dapagliflozin treatment in patients with different stages of type 2 diabetes mellitus: effects on glycaemic control and body weight. Diabetes Obes Metab 12: 510-516.
Visit SAGE journals online http://tae.sagepub.com

@SAGE journals 